\title{
Immune Check-Point Inhibitors in Breast Cancer: Current Evidence and Future Directions
}

\author{
Mosteiro $\mathrm{M}^{1 *}$, Cejuela $\mathrm{M}^{1 *}$ and Pernas $\mathrm{S}^{1,2 *}$ \\ ${ }^{1}$ Department of Medical Oncology, Catalan Institute of \\ Oncology (ICO), L'Hospitalet de Llobregat, Barcelona, \\ Spain \\ ${ }^{2}$ Breast Cancer Group, Institut d'Investigació Biomèdica \\ de Bellvitge (IDIBELL), L’Hospitalet de Llobregat, \\ Barcelona, Spain \\ "Contributed Equally to this Work \\ *Corresponding author: Sonia Pernas, Department \\ of Medical Oncology, Catalan Institute of Oncology, Gran \\ Via de l'Hospitalet 199-203, Spain
}

Received: April 27, 2021; Accepted: J une 02, 2021;

Published: June 09, 2021

\begin{abstract}
Check-point inhibitors have erupted as a treatment option for numerous kinds of neoplasms. Although there have been some achievements, the evidence supporting their use in breast cancer is scarce. Combinations with chemotherapy seem to provide better outcomes, and triple negative is the subtype most likely to benefit from them. New combination strategies are undergoing research to improve these results. Other approaches to determining biomarkers that identify which populations clearly benefit from these therapies are needed. Here, we review the clinical data of the role of immune check-point inhibitors in early and advanced breast cancer and present emerging strategies.

Keywords: Breast cancer; Immunotherapy; Immune checkpoints; PD-L1; Tumor-infiltrating lymphocytes
\end{abstract}

\section{Abbreviations}

BC: Breast Cancer; ICIs: Immune Checkpoint Inhibitors; PD-1: Programmed cell Death protein 1; CTLA-4: Cytotoxic T-LymphocyteAssociated Protein 4; PD-L1: Programmed Death-Ligand 1; PD-L2: Programmed Death-Ligand 2; TILs: Tumor Infiltrating Lymphocytes; TNBC: Triple Negative Breast Cancer; HR: Hormone Receptor; HER2: Human Epidermal Growth Factor Receptor 2; OS: Overall Survival; ORR: Overall Response Rate; PFS: Progression Free Survival; CPS: Combined Positive Score; ITT: Intention To Treat; pCR: Pathological Complete Response; EFS: Event-Free Survival; AEs: Adverse Events; TMB: Tumor Mutational Burden; PR: Partial Response; SD: Stable Disease; T-DM1: Trastuzumab Emtansine; PARPi: Poly-ADP-Ribose-Polymerase inhibitors; DCR: Disease Control Rate; TPS: Tumor Proportion Score

\section{Introduction}

Breast Cancer (BC) is the most common malignancy and the second leading cause of cancer death in women worldwide. There were over 2.1 million newly diagnosed cases in 2018, accounting for one out of four cancer cases in women, and a total of 630,000 deaths [1]. Prognosis in western countries has improved in recent years, due to advances in treatment and earlier detection [2,3]. Nevertheless, metastatic disease is still a deadly illness, and finding new therapeutic strategies is of the utmost importance.

The host immune system has an important role in tumor initiation and progression. Exploiting intrinsic mechanisms of the host immune system to eradicate cancer cells has achieved impressive success. James Allison and Tasuku Honjo developed Immune Checkpoint Inhibitors (ICIs) which have dramatically changed the prognosis of multiple types of neoplasms such as lung cancer and melanoma, among others. Under normal conditions, the immune system uses an inhibitory checkpoint pathway to stop the immune response against pathogens and prevent autoimmune activity. This mechanism is carried out by the Programmed cell Death protein 1 (PD-1) and the Cytotoxic T-Lymphocyte-Associated protein 4 (CTLA-4) which down-regulate and inhibit T-cells by binding to their ligands: Programmed DeathLigand 1 (PD-L1), Programmed Death-Ligand 2 (PD-L2) and CD80/ CD86 [4]. Tumor cells take advantage of this mechanism to create an immunosuppressive microenvironment in which they can hide from the immune system [5]. The anti-PD-1, anti-PD-L1 and anti-CTLA-4 monoclonal antibodies circumvent this immune down-regulation and boost the immune response to tumor cells [6-12].

$\mathrm{BC}$ is a heterogeneous disease with different molecular and clinical features. It has not traditionally been considered a highly immunogenic disease since it is characterized by a relatively low mutation burden in comparison to other neoplasms [13]. Nevertheless, BC immunogenicity is also heterogeneous, with different rates of immune infiltration depending on tumor subtype. The capacity to induce an immune response is also determined by other factors such as tumor neoantigens [14] or PD-L1 expression in the tumor and its microenvironment [15]. Additionally, some genetic mutations such as BRCA1 and BRCA2 result in homologous repair deficiency, which cause more genomic instability and high mutational loads [16]. Triple-Negative Breast Cancers (TNBC) are generally considered more immunogenic than Hormone Receptor (HR)-positive/Human Epidermal Growth Factor Receptor 2 (HER2)negative $\mathrm{BC}$, and differences in immunogenicity exist also among intrinsic molecular subtypes [17].

Tumor microenvironment includes a wide range of immune cells from both the innate and adaptive response. The quantification and morphological evaluation of these immune infiltrates have acquired great transcendence as a prognostic and predictive factor for response. Currently, PD-L1 has been established as the main biomarker for response to ICIs. In BC, it is up-regulated in approximately $20 \%$ $34 \%$ of cases and has been linked to younger patients, high-grade and more aggressive tumors [18]. Tumors infiltrating lymphocytes (TILs) and some of its subpopulations have also been related to ICIs effectiveness. Increased TILs infiltration usually correlates with high PD-L1 expression, especially in TNBC. Among BC subtypes, high PD-L1 expression and TILs are more frequent in HER2-positive and 


\section{TNBC $[19,20]$.}

The aim of this review is to summarize the current evidence of ICIs in both early and advanced BC, as well as review future directions and perspectives.

\section{Triple Negative Breast Cancer}

TNBC represents around $15 \%$ of $\mathrm{BC}$ cases. It is characterized by a lack of estrogen and progesterone receptors and HER2 [21]. It is often related to an earlier age at diagnosis, a more aggressive course, and a worse prognosis with more frequent visceral involvement. Although early-stage TNBC is often associated with high rates of response to chemotherapy, relapse is common and tends to appear in the first 3 years after the treatment [22-24]. Once metastasis occurs, TNBC is incurable, with a median Overall Survival (OS) of only 10-13 months [25-27]. At present, there are no specific treatments other than chemotherapy, but efforts are being made to find new therapeutic approaches for these patients.

Some features make TNBC more likely to respond to immunotherapy than other BC. For that reason, ICIs have mainly been tested in this subtype. It has the highest PD-L1 expression and TILs. In fact, an increased TILs infiltration and high PD-L1 expression have been both associated with better prognosis in early TNBC $[28,29]$. Moreover, TNBC holds a greater mutational load, which is related to higher tumor-specific neoantigens [30]. This may activate more neoantigen-specific $\mathrm{T}$ cells to trigger an anti-tumor response that can be strengthened by ICIs.

Several trials have evaluated therapies with the anti-PD-1 antibody pembrolizumab and the anti-PD-L1 antibodies avelumab, durvalumab and atezolizumab in TNBC.

\section{$\mathrm{ICl}$ as single-agent in metastatic TNBC}

The first trial reporting the clinical benefit of ICIs in TNBC was the KEYNOTE-012 (NCT01848834) which studied pembrolizumab in patients with metastatic TNBC with at least $1 \%$ of PD-L1 expression in either immune or tumor cells. Although most patients had previously been treated (84.4\%), and over $46 \%$ of them had received $\geq 3$ previous lines, the trial showed promising results with an Overall Response Rate (ORR) of 18.5\% [31].

Atezolizumab was also tested in metastatic TNBC in the phase I trial PCD4989g (NCT01375842). Of the 116 total patients included, $58 \%$ had received at least one prior line of treatment. Those who received atezolizumab as first-line therapy with PD-L1 positive tumors presented better ORR, (Table 1) whereas none of the PD-L1 negative patients responded [32].
A further approach was made in the KEYNOTE-086 (NCT02447003), a phase II trial where patients with advanced TNBC were divided into two cohorts according to the treatment previously received in the metastatic setting and PD-L1 expression (Table 1). Cohort A included patients who had received at least one prior treatment regardless of PD-L1 status; while cohort B included only patients in the first line with positive PDL1 expression. In cohort $A$, the ORR was similar in PDL-1 positive and negative tumors. In Cohort B, the ORR was much higher. Progression Free Survival (PFS) was similar in both cohorts but patients in cohort B presented longer OS. These results suggest that pembrolizumab provides more benefits when given in the first line setting and in tumors with positive PD-L1 expression. Investigators evaluated TILS levels in the population included in this study, and correlated it with response to pembrolizumab. Interestingly, the median TILs levels were higher in untreated patients, and those whose tumors had greater stromal TILs showed a better response to immunotherapy $[33,34]$.

Supporting the results of the trial PCD4989g and cohort A of the KEYNOTE-086, avelumab (JAVELIN trial, NCT01772004) showed similar outcomes in terms of ORR, PFS, and OS in pretreated advanced TNBC patients [35].

The phase III KEYNOTE-119 (NCT02555657) trial compared pembrolizumab monotherapy versus investigator-choice chemotherapy (capecitabine, eribulin, gemcitabine or vinorelbine) in pre-treated patients with advanced TNBC. Neither OS nor PFS showed improvement with pembrolizumab in any subgroup. Nevertheless, higher ORR was achieved in patients with combined positive score (CPS) $\geq 10$ and CPS $\geq 1$. In an exploratory analysis, patients with a CPS $\geq 20$ seemed to have lower risk of death in the pembrolizumab arm, with an increase in OS (14.9 vs. 12.5 months), and longer maintained responses, but no statistically significant improvement in PFS (3.4 vs. 2.4 months) [36].

Although immunotherapy was expected to have a significant impact on advanced TNBC, the efficacy shown by ICIs as a single agent has been poor so far. Taking these results together (Table 1), it is possible to gather two major insights. First, patients with PD-L1 positive tumors are more likely to obtain clinical benefit. Second, ICIs in monotherapy provide a higher response rate in earlier lines (ORR of $20-25 \%$ vs. $5-8 \%$ in later lines).

\section{$\mathrm{ICl}$ in combination with chemotherapy in metastatic TNBC}

Chemotherapy can decrease the number of immunosuppressive cells and up-regulate pro-inflammatory cytokines in the tumor environment. Moreover, when tumor cells are destroyed by chemotherapy, they release molecules such as ATP, calreticulin or

Table 1: Phase I-II clinical trials assessing checkpoint inhibitors as single-agent in metastatic TNBC.

\begin{tabular}{|c|c|c|c|c|c|c|c|c|c|}
\hline Trial & Phase & $\mathrm{ICl}$ & $\%$ PD-L1 + population & Prior lines & ORR & ORR PD-L1 + vs. PD-L1 - & mDoR & mPFS & mos \\
\hline KEYNOTE-012 & 1 & Pembrolizumab & $100 \%$ & Any & $18.50 \%$ & - & NR & $1.9 \mathrm{~m}$ & $11.2 \mathrm{~m}$ \\
\hline JAVELIN & 1 & Avelumab & $68.80 \%$ & $1-3$ & $5.20 \%$ & $22.2 \%$ vs. $2.6 \%$ & NR & $1.5 \mathrm{~m}$ & $9.2 \mathrm{~m}$ \\
\hline PCD4989g & 1 & Atezolizumab & $78 \%$ & $\begin{array}{c}0 \\
\geq 1\end{array}$ & $\begin{array}{c}24 \% \\
6 \%\end{array}$ & $12 \%$ vs. $0 \%$ & $21 \mathrm{~m}$ & $1.4 \mathrm{~m}$ & $8.9 \mathrm{~m}$ \\
\hline KEYNOTE-086 A & II & Pembrolizumab & $61.80 \%$ & $\geq 1$ & $5.30 \%$ & $5.7 \%$ vs. $0 \%$ & NR & $2.0 \mathrm{~m}$ & $9.0 \mathrm{~m}$ \\
\hline KEYNOTE-086 B & II & Pembrolizumab & $100 \%$ & 0 & $21.50 \%$ & - & $10.4 \mathrm{~m}$ & $2.1 \mathrm{~m}$ & $18.0 \mathrm{~m}$ \\
\hline
\end{tabular}

ICl: Immune Check-Point Inhibitor; PD-L1: Programmed Death-Ligand 1; ORR: Overall Response Rate; mDoR: Median Duration of Response; mPFS: Median Progression Free Survival; mOS: Median Overall Survival; NR: Not Reached; m: Months. 
Table 2: Phase III clinical trials assessing checkpoints inhibitors plus chemotherapy in TNBC

\begin{tabular}{|c|c|c|c|c|c|c|c|c|c|c|c|}
\hline Trial & $\mathbf{n}$ & \multicolumn{2}{|c|}{$\begin{array}{l}\text { Primary } \\
\text { Endpoint/s }\end{array}$} & \multicolumn{2}{|c|}{ Experimental Arm } & \multicolumn{4}{|c|}{ Control Arm } & \multicolumn{2}{|c|}{ Results } \\
\hline \multicolumn{12}{|c|}{ Metastatic Setting } \\
\hline $\begin{array}{l}\text { KEYNOTE } \\
355\end{array}$ & 847 & \multicolumn{2}{|c|}{ PFS and OS } & \multicolumn{2}{|c|}{$\begin{array}{l}\text { Nab-paclitaxel/Paclitaxel/Carboplatin plus } \\
\text { gemcitabine + Pembrolizumab }\end{array}$} & \multicolumn{4}{|c|}{$\begin{array}{l}\text { Nab-paclitaxel /Paclitaxel/Carboplatin plus } \\
\text { gemcitabine + Placebo }\end{array}$} & \multicolumn{2}{|c|}{$\begin{array}{l}\text { CPS }>10 \text { mPFS: } 9.7 \text { vs. } \\
5.6 \mathrm{~m} \\
H R=0.65 ; p=0.0012\end{array}$} \\
\hline $\begin{array}{l}\text { IMpassion } \\
130\end{array}$ & 902 & \multicolumn{2}{|c|}{ PFS and OS } & \multicolumn{2}{|c|}{ Nab-paclitaxel + Atezolizumab } & \multicolumn{4}{|c|}{ Nab-paclitaxel + Placebo } & \multicolumn{2}{|c|}{$\begin{array}{c}\text { PD-L1+ mPFS: } 7.2 \text { vs. } 5.5 \mathrm{~m} \\
\quad H R=0.62 ; p<0.001\end{array}$} \\
\hline $\begin{array}{l}\text { IMpassion } \\
131\end{array}$ & 651 & \multicolumn{2}{|c|}{ PFS } & \multicolumn{2}{|c|}{ Paclitaxel + Atezolizumab } & \multicolumn{4}{|c|}{ Paclitaxel + Placebo } & \multicolumn{2}{|c|}{$\begin{array}{c}\text { PD-L1+ mPFS: } 6.0 \text { vs. } 5.7 \mathrm{~m} \\
\text { HR }=0.82 ; p=0.20\end{array}$} \\
\hline \multicolumn{12}{|c|}{ Neoadjuvant Setting } \\
\hline $\begin{array}{l}\text { KEYNOTE } \\
522\end{array}$ & 602 & \multicolumn{2}{|c|}{$\begin{array}{l}\text { pCR rate and } \\
\text { EFS }\end{array}$} & \multicolumn{2}{|c|}{$\begin{array}{l}\text { Carboplatin + Paclitaxel + Pembrolizumab } \\
\text { followed by AC + Pembrolizumab }\end{array}$} & \multicolumn{4}{|c|}{$\begin{array}{c}\text { Carboplatin + Paclitaxel + Placebo followed } \\
\text { by AC+ Placebo }\end{array}$} & \multicolumn{2}{|c|}{$\begin{array}{l}\text { pCR: } 64.8 \% \text { vs. } 51.2 \% \\
\text { p }<0.001\end{array}$} \\
\hline $\begin{array}{l}\text { IMpassion } \\
031\end{array}$ & 333 & \multicolumn{2}{|c|}{$\mathrm{pCR}$ rate } & \multicolumn{2}{|c|}{$\begin{aligned} \text { Nab-paclitaxel } & + \text { Atezolizumab followed by ACdd } \\
& + \text { Atezolizumab }\end{aligned}$} & \multicolumn{4}{|c|}{$\begin{array}{l}\text { Nab-paclitaxel }+ \text { Placebo followed by ACdd } \\
+ \text { + Placebo }\end{array}$} & \multicolumn{2}{|c|}{$\begin{array}{l}\text { pCR: } 58 \% \text { vs. } 41 \% \\
p=0.0044\end{array}$} \\
\hline NeoTRIP & 280 & \multicolumn{2}{|c|}{ EFS } & \multicolumn{2}{|c|}{ Carboplatin + Nab-paclitaxel + Atezolizumab } & \multicolumn{4}{|c|}{ Carboplatin + Nab-paclitaxel } & \multicolumn{2}{|c|}{$\begin{array}{c}\text { pCR: } 43.5 \% \text { vs. } 40.8 \% \\
\text { not significant }\end{array}$} \\
\hline \multicolumn{12}{|c|}{$\begin{array}{l}\text { PFS: Progression Free Survival; OS: Overall Survival; CPS: Combined Positive Score; mPFS: Median Progression Free Survival; m: Months; HR: Hazard Ratio; } \\
\text { pCR: Pathological Complete Response; EFS: Event Free Survival; AC: Doxorubicin and Cyclophosphamide; ACdd: Doxorubicin and Cyclophosphamide Dose Dense. }\end{array}$} \\
\hline \multirow{2}{*}{\multicolumn{2}{|c|}{ Trial }} & \multirow{2}{*}{ Phase } & \multirow{2}{*}{$\mathbf{n}$} & \multirow{2}{*}{$\mathrm{ICI}$} & & & & & & & \\
\hline & & & & & Anti-HER2 Ther & ару & PD-L1 & PD-L1+ & & PD-L1- & PD-L1 ${ }^{+}$ \\
\hline CCTGIND. & 29 & $\mathrm{lb}$ & 15 & Durvalumab & Trastuzumab & & $0 \%$ & - & & $1.35 \mathrm{~m}$ & - \\
\hline JAVELII & & I & 26 & Avelumab & Trastuzumab & & $0 \%$ & - & & - & - \\
\hline PANACE & & |b-II & 58 & Pembrolizumab & Trastuzumab & & $0 \%$ & $15 \%$ & & $2.5 \mathrm{~m}$ & $2.7 \mathrm{~m}$ \\
\hline KATE 2 & & II & 202 & Atezolizumab/placebo & T-DM1 & & 39 vs. $50 \%$ & 54 vs. $33 \%$ & 6.8 & vs. $8.2 \mathrm{~m}$ & 8.5 vs. $4.1 \mathrm{~m}$ \\
\hline NCT03523 & 572 & $\mathrm{lb}$ & 48 & Nivolumab & Trastuzumab-derux & xtecan & & & & & \\
\hline
\end{tabular}

ICl: Immune Check-Point Inhibitor; PD-L1: Programmed Death-Ligand 1; ORR: Overall Response Rate; PFS: Progression Free Survival; m: Months.

HMGB1 that activate dendritic cells and work as neoantigens to stimulate T-cells [37]. This process is called immunogenic death, and can enhance the effect of ICI. Based on this evidence, some clinical trials have assessed the combination of chemotherapy with immunotherapy in BC (Table 2).

The phase III KEYNOTE-355 (NCT02819518) compared the efficacy of several chemotherapy regimens (nab-paclitaxel, paclitaxel, or carboplatin plus gemcitabine) in combination with pembrolizumab or placebo as first line treatment in patients with advanced TNBC. PD-L1 expression was tested by Dako 22C3 pharmDx assay, used 22C3 antibody and it is calculated by the number of PD-L1-positive cells (tumor cells, lymphocytes, and macrophages) divided by the total number of tumor cells. Co-primary endpoints were PFS and OS by PDL-1 tumor expression (CPS $\geq 10$ or CPS $\geq 1$ ) and Intention to Treat (ITT) population. Although the combination only showed statistically significant benefit for PFS in the patients with CPS $\geq 10$, a tendency toward higher pembrolizumab efficacy with PD-L1 enrichment was observed. The hazard ratio for PFS favored pembrolizumab despite the chemotherapy background [38]. OS follow-up is still ongoing. Based on PFS benefit, the FDA approved pembrolizumab with chemotherapy in the first line of advanced TNBC with PD-L1 CPS $\geq 10$ [39].

Pembrolizumab was also tested in combination with eribulin in the single arm phase I study ENHANCE-1 (NCT02513472). This clinical trial enrolled patients with advanced TNBC and $\leq 2$ prior lines of treatment. The ORR was $23.4 \%$, and similar to the studies in monotherapy, the ORR was higher in non-previously treated and PD-L1 positive patients [40].
The first study evaluating atezolizumab with chemotherapy was a phase Ib trial (NCT01633970) in which 33 patients received atezolizumab in combination with nab-paclitaxel. The treatment provided benefit in terms of ORR of $39.4 \%$, with a PFS and OS of 5.5 months (95\% CI, 5.1-7.7 months) and 14.7 months (95\% CI, 10.1-not able to be estimated), respectively [41]. Following these positive results, the phase III trial IMpassion130 (NCT0242589) was conducted. It included advanced TNBC patients with no prior treatment regardless of $\mathrm{PD}-\mathrm{L} 1$ status. $\mathrm{PD}-\mathrm{L} 1$ expression was assessed by VENTANA SP142 PD-L1 clone on immune cells, and levels over $1 \%$ were considered positive. At the first interim analysis with a median follow up of 12.9 months, atezolizumab improved the PFS slightly within 2 months in the ITT and the PDL-1 positive population [42]. At a median follow-up of 18-months, the median OS in the ITT population was 21.0 months with atezolizumab, and 18.7 months with placebo. Median OS was even longer (25 months) in the PD-L1 positive group [43]. Succeeding these achievements, the FDA and the EMA approved the use of atezolizumab $(840 \mathrm{mg}$ iv. on day 1 and day 15 of every 28 -day cycle) in combination with nab-paclitaxel (100mg/ $\mathrm{m}^{2}$ iv. on days 1,8 and 15$)$ in the first line treatment for patients with advanced TNBC and positive PD-L1 expression ( $\geq 1 \%)$.

The Impassion 131 trial studied the combination of paclitaxel plus either atezolizumab or placebo in advanced TNBC. Although the design was similar to the IMpassion 130, it was a negative trial and neither ITT nor PDL1-positive population obtained benefit from the combination [44]. The contradictory outcomes in the IMpassion 130 and the IMpassion 131 were deceptive. This was initially related to divergences in the patients included in the two trials. Nevertheless, this theory was rejected because a subgroup analysis of the population 
Table 4: Ongoing phase III clinical trials with checkpoint inhibitors in metastatic breast cancer.

\begin{tabular}{|c|c|c|c|c|c|c|}
\hline Trial & Setting & $\mathbf{n}$ & Experimental Arm & Control Arm & $\begin{array}{l}\text { Combinatory drug } \\
\text { MoA }\end{array}$ & $\begin{array}{l}\text { Primary } \\
\text { endpoint }\end{array}$ \\
\hline $\begin{array}{l}\text { NCT04732598 } \\
\text { (AMBITION) }\end{array}$ & $1^{\text {st }}$ line HER2 negative & 280 & $\begin{array}{c}\text { Bevacizumab + Paclitaxel + } \\
\text { Atezolizumab }\end{array}$ & Bevacizumab + Paclitaxel & antiVEGF & PFS \\
\hline NCT04177108 & $1^{\text {st }}$ line TNBC & 242 & Paclitaxel + Placebo & $\begin{array}{c}\cdot \text { Paclitaxel + Ipatasertib + Atezolizumab } \\
\cdot \text { Paclitaxel + Ipatasertib + Placebo } \\
\cdot \text { Paclitaxel + Atezolizumab + Placebo }\end{array}$ & $\mathrm{AKTi}$ & $\begin{array}{l}\text { PFS } \\
\text { OS }\end{array}$ \\
\hline $\begin{array}{l}\text { NCT04191135 } \\
\text { (MK-7339-009/ } \\
\text { KEYLYNK-009) }\end{array}$ & $1^{\text {st }}$ line TNBC & 932 & $\begin{array}{c}\text { Carboplatin + Gemcitabine + } \\
\text { Peembrolizumab }\end{array}$ & $\begin{array}{l}\text { Carboplatin + Gemcitabine + } \\
\text { Pembrolizumab followed by } \\
\text { pembrolizumab + Olaparib }\end{array}$ & PARPi & $\begin{array}{l}\text { PFS } \\
\text { OS }\end{array}$ \\
\hline $\begin{array}{c}\text { NCT04148911 } \\
\text { (EL1SSAR) }\end{array}$ & $1^{\text {st }}$ line TNBC & 280 & $\begin{array}{l}\text { Paclitaxel + Atezolizumab } \\
\text { (per investigator choice) }\end{array}$ & Nab-paclitaxel + Atezolizumab & - & Safety \\
\hline $\begin{array}{c}\text { NCT03199885 (NRG- } \\
\text { BR004) }\end{array}$ & $1^{\text {st }}$ line HER2 positive & 600 & $\begin{array}{l}\text { Pertuzumab + Trastuzumab } \\
+ \text { Paclitaxel + Atezolizumab }\end{array}$ & $\begin{array}{c}\text { Pertuzumab + Trastuzumab + } \\
+ \text { +Paclitaxel + Placebo }\end{array}$ & $\begin{array}{l}\text { dual HER2 } \\
\text { blockade }\end{array}$ & PFS \\
\hline NCT04740918 (KATE3) & $\begin{array}{l}\text { up to } 3^{\text {rd line HER2 }} \\
\text { positive and PD-L1 } \\
\text { positive BC }\end{array}$ & 350 & TDM1 + Atezolizumab & TDM1 + Placebo & $\begin{array}{l}\text { HER2-targeted } \\
\text { antibody-drug } \\
\text { conjugate }\end{array}$ & $\begin{array}{l}\text { PFS } \\
\text { OS }\end{array}$ \\
\hline
\end{tabular}

MoA: Mechanism of Action; PARPi: Poly-ADP-Ribose-Polymerase Inhibitors; VEGF: Vascular Endothelial Growth Factor, AKTi: AKT Inhibitor; PARPi: Poly-ADPRibose-Polymerase Inhibitors; PFS: Progression Free Survival; OS: Overall Survival; mTNBC: Metastatic Triple Negative Breast Cancer; PD-L1: Programmed DeathLigand 1.

Table 5: Ongoing phase III clinical trials with checkpoint inhibitors in early-stage breast cancer.

\begin{tabular}{|c|c|c|c|c|c|}
\hline Trial & Setting & $\mathbf{n}$ & Experimental Arm & Control Arm & Primary endpoint \\
\hline $\begin{array}{l}3726879 \text { (IMpassion } \\
\text { 050) }\end{array}$ & $\begin{array}{l}\text { HER2-positive } \\
\text { Neoadjuvancy }\end{array}$ & 453 & $\begin{array}{l}\text { AC + atezolizumab followed by Paclitaxel + } \\
\text { Pertuzumab + Trastuzumab + atezolizumab }\end{array}$ & $\begin{array}{l}\text { AC + placebo followed by Paclitaxel + } \\
\text { Pertuzumab + Trastuzumab + placebo }\end{array}$ & $\begin{array}{l}\mathrm{PCR} \text { in the ITT and } \\
\text { PDL1+ population }\end{array}$ \\
\hline $\begin{array}{l}\text { NCT03595592 } \\
\text { (APTneo) }\end{array}$ & $\begin{array}{l}\text { HER2-positive } \\
\text { Neoadjuvancy }\end{array}$ & 650 & $\begin{array}{c}\cdot A C+\text { atezolizumab followed by HTCP + } \\
\text { atezolizumab } \\
\cdot \mathrm{HTCP}+\text { atezolizumab }\end{array}$ & HTCP & EFS \\
\hline NCT02954874 & $\begin{array}{l}\text { TNBC with } \\
\text { residual } \\
\text { disease. } \\
\text { Ajduvancy }\end{array}$ & 1050 & Pembrolizumab & Observation & $\begin{array}{l}\text { iDFS } \\
\text { Severity of fatigue } \\
\text { Physical function }\end{array}$ \\
\hline NCT03281954 & TNBC & 1520 & $\begin{array}{l}\text { Paclitaxel + carboplatin + atezolizumab followed } \\
\text { by } A C+\text { atezolizumab and adjuvant atezolizumab }\end{array}$ & $\begin{array}{c}\text { Paclitaxel + carboplatin + placebo } \\
\text { followed by AC + placebo and adjuvant } \\
\text { placebo }\end{array}$ & $\begin{array}{l}\text { pCR } \\
\text { EFS }\end{array}$ \\
\hline $\begin{array}{l}\text { NCT03498716 } \\
\text { (IMpassion030) }\end{array}$ & TNBC & 2300 & $\begin{array}{c}\text { Paclitaxel + atezolizumab followed by EC/AC + } \\
\text { atezolizumab and adjuvant atezolizumab }\end{array}$ & Paclitaxel followed by EC/AC & iDFS \\
\hline $\begin{array}{l}\text { NCT03197935 } \\
\text { (IMpassion031) }\end{array}$ & TNBC & 324 & $\begin{array}{c}\text { Nab-paclitaxel + atezolizumab followed by } \mathrm{AC}+ \\
\text { atezolizumab and adjuvant atezolizumab }\end{array}$ & $\begin{array}{l}\text { Nab-paclitaxel + placebo followed by } \\
\mathrm{AC}+\text { placebo and adjuvant placebo }\end{array}$ & $\begin{array}{l}\text { PCR in the ITT and } \\
\text { PD-L1 positive } \\
\text { population }\end{array}$ \\
\hline $\begin{array}{l}\text { NCT03725059 } \\
\text { (MK-3475-756/ } \\
\text { KEYNOTE-756) }\end{array}$ & $\mathrm{HR}+$ & 1140 & $\begin{array}{c}\text { Paclitaxel + pembrolizumab followed by AC/ } \\
\mathrm{EC}+\text { pembrolizumab and adjuvant + ET + } \\
\text { pembrolizumab }\end{array}$ & $\begin{array}{l}\text { Paclitaxel + placebo followed by AC/EC } \\
\text { + placebo and adjuvant ET + placebo }\end{array}$ & $\begin{array}{l}\text { PCR } \\
\text { EFS }\end{array}$ \\
\hline
\end{tabular}

PD-L1: Programmed Death-Ligand 1; pCR: Pathological Complete Response; ITT: Intention to Treat; EFS: Event Free Survival; iDFS: Invasive Disease-Free Survival; AC: Doxorubicin and Cyclophosphamide; HTCP: Herceptin + Paclitaxel + Carboplatin + Pertuzumab; EC: Epirubicin and Cyclophosphamide; ET: Endocrine Therapy.

in the KEYNOTE-355 and the IMpassion130 showed that those experiencing greater benefit had positive PD-L1 expression, metastasis at the onset of the illness, disease free survival over 6 months and no prior chemotherapy in the early stage of the disease. These characteristics were equally present in the IMpassion 130 and IMpassion131 participants. Another explanation for this was a possible deleterious effect of the corticosteroid premedication given with paclitaxel. Nevertheless, a subgroup analysis of the KEYNOTE-355 was recently presented. In contrast to IMpassion131 results, patients treated with paclitaxel obtained a benefit in PFS with the addition of pembrolizumab [39]. Based on this information, definitive conclusions cannot be reached yet.

It should be pointed out that in all studies testing the combination of chemotherapy and ICIs, safety and toxicity profiles were similar to those observed with immunotherapy or chemotherapy alone. However, the increasing number of immune-related Adverse Events (AEs) in the combination arms stands out, with $25.6 \%$ of patients experiencing an immune-related adverse event in any grade, including $5.2 \%$ of grade $3-5$ [38,40-44].

\section{$\mathrm{ICl}$ in early-stage TNBC}

TNBC has a worse prognosis than the other subtypes, even in early stages. Neoadjuvant chemotherapy is the preferred therapeutic approach in most of the cases. Pathological Complete Response (pCR) is achieved in one third of patients with stage II-III BC receiving an anthracycline and taxane-based neoadjuvant chemotherapy. With the addition of platinum compounds to neoadjuvant chemotherapy, about one half of patients achieve a pCR [45-51]. pCR is a surrogate marker of long-term survival outcomes, and it is a valid endpoint for accelerated drug approval. In early BC, ICI have mainly been tested in association with chemotherapy, with the goal of achieving higher pCR rates and reducing rates of recurrence disease [51-53].

Two early trials tested pembrolizumab added to chemotherapy in the neoadjuvant setting of high risk TNBC, and both described similar pCR rates in favor of the combination. First, the I-SPY2 trial (NCT01042379) tested pembrolizumab with weekly paclitaxel and anthracyclines in early HER-2 negative BC (TNBC cohort and $\mathrm{HR}+$ cohort), regardless PD-L1 status. In the TNBC cohort, pembrolizumab-chemotherapy provided a pCR rate of $60 \%$ vs. $22 \%$ 
in the control arm [54]. The KEYNOTE-173 (NCT02622074) was a single arm phase Ib study in which pembrolizumab was added to six different chemotherapy regimens. In this trial, higher rates of PDL1 expression and stromal TILs levels were associated with a higher probability of achieving a PCR [55].

Following these encouraging results, several phase III trials evaluated the role of immunotherapy in combination with neoadjuvant chemotherapy in TNBC (Table 2). The KEYNOTE-522 trial (NCT03036488) randomized patients with high risk TNBC to receive weekly paclitaxel plus carboplatin and anthracyclines either plus pembrolizumab or placebo. Pembrolizumab was continued in the adjuvant setting to complete one year treatment. The two primary endpoints, pCR and Event-Free Survival (EFS) were improved in favor of the combination arm. Notably, unlike previous findings in the metastatic setting and KEYNOTE 173, the benefit of the anti PDL1 drug in this setting was not related to PD-L1 expression [56].

The phase III IMpassion031 (NCT03197935) trial also studied the combination of atezolizumab and chemotherapy in the neoadjuvant setting. Chemotherapy regimen consisted of weekly nab-paclitaxel for 12 weeks, followed by 4 cycles of dose dense doxorubicin plus cyclophosphamide. The pCR rate in the ITT population was 58\% vs. $41 \%$ in favor of the atezolizumab arm. Although atezolizumab provided a higher $\mathrm{pCR}$ rate in the $\mathrm{PD}-\mathrm{L} 1$ positive population $(69 \%$ vs. $49 \%$ ), patients without PD-L1 expression also showed a higher $\mathrm{pCR}$ rate with atezolizumab ( $48 \%$ vs. 34\%) [57].

The randomized phase II study GeparNuevo investigated the addition of durvalumab/placebo to neoadjuvant chemotherapy based on nab-paclitaxel followed by dose-dense epirubicin plus cyclophosphamide. The pCR rate in the durvalumab arm for PDL1 positive tumors was $58.0 \%$ vs. $44.4 \%$ in the PD-L1 negative group, while in the placebo arm pCR was $50.7 \%$ vs. $18.2 \%$, but these differences were not statistically significant. This trial was also designed to identify potential biomarkers of response to immunotherapy. The only biomarker that predicted benefit with the combination was the increase of intratumoral TILs following the window-phase in the durvalumab arm. In the placebo arm, the change of intratumoral TILs did not predict pCR. PD-L1 positivity was also associated with an increased pCR rate in both arms [58].

The NeoTRIP study (NCT02620280) is a phase III clinical trial that compared carboplatin (AUC2) plus nab-paclitaxel with atezolizumab or placebo as neoadjuvant treatment in high-risk TNBC. After surgery, 4 cycles of anthracycline-based regimen were administered as per investigator choice. The primary endpoint was EFS at 5 years, and pCR was a secondary endpoint. Results for EFS analysis are still not available. In contrast to results from other trials, pCR rate was not significantly higher either in the ITT population or in patients with PD-L1 positive tumors [59]. The lack of benefit for atezolizumab was initially related to the population characteristics. In a subgroup analysis of the KEYNOTE-522 and the IMpassion031, nodal involvement was the only clinical characteristic related to greater benefit with the combination. The KEYNOTE-522 showed a difference in pCR rate of $20.5 \%$ vs. $6.3 \%$, whereas the IMpassion 031 showed a difference of $26.6 \% v s .8 .8 \%$ in favor of patients with nodal involvement. Nevertheless, the proportion of patients with nodal involvement was higher in the NeoTRIP study which contradicts this theory. Differences in the chemotherapy backbone could be responsible for the lack of benefit observed with atezolizumab in the NeoTRIP study. The phase II TONIC trial compared the ORR with nivolumab after several induction schemes (radiotherapy, cyclophosphamide, cisplatin and doxorubicin). In the overall population, the ORR was $20 \%$, but best responses were seen following cisplatin and doxorubicin induction, with ORR of $23 \%$ and $35 \%$, respectively [60]. These results support the idea that the lack of anthracyclines might cause the negative outcomes of the NeoTRIP trial.

As observed in the metastatic setting, the combination of ICIs and chemotherapy was well tolerated with a similar safety profile apart from mild grade 1 and 2 immune mediated toxicities. The most frequent toxicity related to ICIs was thyroid dysfunction and transaminitis. Nevertheless, around $14-15 \%$ of patients experienced immune-mediated AE side effects, some of which were potentially serious (above 0.1\%) [54-60]. This fact is especially important in the setting of a curable disease. Training to identify and treat these serious AEs is crucial.

\section{ICI in Hormone Receptor-Positive Breast Cancer}

Hormone Receptor Positive BC is the most prevalent subtype, accounting for $60-65 \%$ of all BC cases. Luminal disease has an immunologically cold nature. It is associated with lower rates of PDL1 expression and TILs as well as less genomic instability and Tumor Mutational Burden (TMB) [61-64]. ICIs have also been tested in the metastatic and early setting, with less encouraging results than those observed in TNBC.

Avelumab and pembrolizumab have been studied as singleagent in the metastatic disease. Avelumab only achieved an ORR of $2.8 \%$ (95\% CI; 0.3-9.7\%) in the HR+ BC cohort of the JAVELIN trial [35]. The KEYNOTE-028 (NCT02054806) included 25 patients with metastatic HR+ BC with positive PD-L1 expression. Every patient had already received at least one prior line of chemotherapy, and $48 \%$ were heavily pretreated ( $\geq 5$ prior lines). Pembrolizumab activity was modest with 3 patients achieving a Partial Response (PR) (12\%) and 4 (16\%) Stable Disease (SD). Median PFS (1.8 months) and median OS were low, with median response duration of 20 weeks (range, 15.737.4 weeks) [65].

The combination of pembrolizumab with eribulin in advanced $\mathrm{HR}+\mathrm{BC}$ was evaluated in a randomized phase II clinical trial (NCT03051659). The primary end point of this study was PFS. After a median follow up of 10.5 months, no benefit in PFS was observed either in the ITT population (4.1 months in the combination arm vs. 4.2 months in the single-agent eribulin arm) nor in the PD-L1 positive population (4.2 months vs. 4.3 months). No statistically significant differences in the ORR were observed either. The ORR was 27\% (95\% CI, $14.9 \%-42.8 \%$ ) for patients receiving eribulin with pembrolizumab and $34 \%$ (95\% CI, 20.5\%-49.9\%) for patients receiving eribulin alone. Moreover, there were no complete responses in either arm, and no significant differences in duration of response (1.5 months for the combination arm vs. 2.1 months). The OS data are still immature, but there are no statistically significant differences so far [66].

Treatment with ICI neither in monotherapy nor in combination 
has been able to improve the outcomes for patients with advanced HR+ disease. In contrast, results of the combination of pembrolizumab with chemotherapy as neoadjuvant treatment explored in the I-SPY2 clinical trial are encouraging. Patients with $\mathrm{HR}+\mathrm{BC}$ treated with the combination achieved a pCR rate of $30 \%$, that doubled the pCR of $13 \%$ observed in the chemotherapy alone arm [54].

\section{ICI in HER2-Positive Breast Cancer}

The HER2 receptor is amplified or overexpressed in 15\%-20\% of BC. The introduction of anti-HER2 targeted therapies such as trastuzumab, pertuzumab, and trastuzumab emtansine (T-DM1) has significantly improved the prognosis of both the early and advanced settings [67-70]. Despite this breakthrough, nearly all metastatic HER2-positive BC patients eventually progress on antiHER2 therapy. In this context, ICIs have mainly been tested in combination with anti-HER2 directed therapy. Preclinical studies in immunocompetent mice showed promising results in favor of the addition of ICIs to the antiHER2 blockade [71]. Nevertheless, these data have not been consistent with the clinical evidence (Table 3).

The combination of the anti-HER2 drug trastuzumab plus durvalumab (CCTGIND.229, NCT02649686) and avelumab (JAVELIN, NCT01772004) in metastatic disease has been tested in two phase I trials, failing to achieve meaningful clinical benefits $[35,72]$. Nevertheless, in the phase II PANACEA trial (NCT02129556) pembrolizumab seemed to offer some benefit in terms of PFS and ORR when added to trastuzumab in the PD-L1 positive subgroup population [73].

In the phase II KATE2 trial (NCT02924883) patients were randomized to receive either T-DM1 plus atezolizumab or T-DM1 plus placebo. After 8.4 months of follow up, atezolizumab improved the median PFS, in the ITT population and PD-L1 positive subgroup, but none were statistically significant [74]. The phase III KATE3, which only includes HER2-positive and PD-L1 positive population, is ongoing (Table 4).

The combination of the antibody drug conjugate trastuzumabderuxtecan with nivolumab, was explored in a phase Ib clinical trial (NCT03523572) recently presented. This trial included not only patients with HER2-positive BC but also with HER2-low expression. The combination demonstrated antitumor activity in both HER2positive and HER2-low breast cancer patients with an ORR of 59.4\% and $37.5 \%$, respectively. Although the safety profile was generally manageable, interstitial lung disease was reported in the $10.4 \%$ of the patients [75].

Results in HER2-positive advanced BC suggest that the benefit from ICI in terms of ORR and/or PFS may be restricted to patients with PD-L1-positive disease. However, research is ongoing to enhance immune activation in HER2-positive breast cancer and to better identify which patients can benefit from it.

\section{Future Directions}

The important benefits of immunotherapy in other cancer types should encourage finding a role for these treatments in BC. Other drugs such as nivolumab, ipilimumab, tremelimumab or spartalizumab are also under study.

Since immunotherapy as a single-agent has not shown significant benefit, the majority of the pipelines are focused on testing combinations of ICI with other agents (Table 4 and 5). Not only different types of chemotherapy, but also cryoablation, radiotherapy, oncolytic virus or targeted therapies are being studied in combination to ICI. Some ICI combinations are being tested, too. The aim of all these strategies is to make $\mathrm{BC}$ more immunogenic and enhance the host immune response. Window of opportunity trials are especially interesting because they allow for an appreciation of the molecular changes in the tumor following short course treatment, and consequently learn from their molecular biology.

Currently, oncology is moving towards a precision medicine where targeted therapies and their combination are becoming crucial. Combinations of ICIs with other targeted therapies are being assessed in many clinical trials. Preclinical models have shown that Poly - ADP - Ribose - Polymerase inhibitors (PARPi) and anti-PD-1 antibodies may have synergistic anti-tumor activity [76]. The TOPACIO trial (NCT02657889) evaluated the combination of niraparib with pembrolizumab in patients with advanced TNBC regardless of the presence of a germline mutation in BRCA and PD-L1 expression. Among the 47 patients who could be evaluated for response, ORR was $21 \%$, with a Disease Control Rate (DCR) of $49 \%$. Response rate was considerably higher in PD-L1 positive patients $(32 \% v s .14 \%)$ and in BRCA mutated patients compared with BRCA wild type (47\% vs. $11 \%)$. The clinical activity of the combination was more pronounced in patients with germline BRCA mutation than in those with PD-L1 positive tumors [77].

The PI3K/AKT/mTOR pathway induces a transcriptional program that promotes immune suppression during tumor growth [78]. It has proven to have an important role in $\mathrm{BC}$ within endocrine resistance, so the combination of ICIs with PI3K, AKT and mTOR inhibitors is also being explored in clinical trials. A phase Ib study (NCT03800836) in which untreated patients with metastatic TNBC were assigned to a taxane (either paclitaxel or nab-paclitaxel) combined with the AKT inhibitor Ipatasertib and atezolizumab showed preliminary results with an impressive ORR of 73\%, regardless PD-L1 expression [79].

Identifying those patients who may benefit from ICI is essential. So far, the main biomarker associated with ICIs benefit has been PD-L1 expression. However, there is no standardized method for measurement, and no clear cutoff of positivity has been defined. The studies with pembrolizumab used the CPS score, measured with Dako 22C3 pharmDx assay, while the studies with atezolizumab used the tumor proportion score (TPS) measured with the VENTANA PD-L1 (SP142) IHC assay. However, these methods are not always consistent [80], and have shown a marked variability among observers. Moreover, it has even been proven that PD-L1 expression differ from the primary and the metastatic specimens.

TILs include different cell types, with T-cells being the most common, but with variable proportions of natural killer cells, dendritic cells, macrophages, and B-cells. As referred before, the prevalence of TILs depends not only on tumor subtype, but also on the stage of the disease, and on metastases sites (with lungs showing the highest degree of TIL infiltration, and the liver and skin the lowest). Early disease shows the greatest rate of TILs infiltration which seems to weaken as the tumor progresses to metastatic disease and across successive lines of treatment. This has been described 
as a tumoral mechanism of avoiding surveillance in which it turns into an inert phenotype. In contrast to early stages, where TILs have been related to better prognosis and sensitivity to chemotherapy (specially in TNBC), in the metastatic setting there is yet much to do. Deeper evaluation integrating both TILs quantity and quality (cells subpopulations), is urgently needed to establish the role of this biomarker [19,20].

Nevertheless, it is important to emphasize that other characteristics such as TMB [81], epigenetic signatures or some clinical features, may also influence the immune checkpoint blockade response. Therefore, future perspectives in biomarkers should cover all these characteristics to integrate them as a prognostic factor and a predictive tool of response [82-86].

\section{Conclusions}

Although the majority of clinical trials have come up with negative results, they show a tendency toward clinical benefit in certain subgroups of patients. Best achievements have been observed with combinations of chemotherapy and immunotherapy, especially in the first line of metastatic TNBC where the FDA approved pembrolizumab with chemotherapy in the first line of advanced TNBC with PD-L1 CPS $\geq 10$. The FDA and EMA also authorized atezolizumab in combination with nab-paclitaxel in patients with PD-L1 positive tumors. In contrast to TNBC, currently there is no approved approach with immunotherapy in patients with HRpositive or HER2 positive BC.

Promising results have been obtained with ICIs in combination with chemotherapy in early-stage BC, especially in TNBC with nodal involvement and PD-L1 positive expression. Patients diagnosed with early TNBC already achieve a high rate of pCR with only chemotherapy, and the addition of ICIs can increase the pCR rate but also the probability of potential serious AEs. Window of opportunity studies could assume a crucial role in identifying new biomarkers to select patients who benefit the most from this strategy.

\section{Conflicts of Interest}

SP has received travel and accommodation grant from Novartis outside of the submitted work and advisor/consultant role for AstraZeneca, Daiichi-Sankyo, Polyphor, Novartis, SeattleGenetics, Eisai, Pierre-Fabre and Roche.

\section{Acknowledgements}

We thank CERCA Programme/Generalitat de Catalunya for institutional support.

\section{References}

1. Bray F, Ferlay J, Soerjomataram I, Siegel RL, Torre LA, Jemal A. Global cancer statistics 2018: GLOBOCAN estimates of incidence and mortality worldwide for 36 cancers in 185 countries. CA Cancer J Clin. 2018; 68: 394424.

2. Autier P, Boniol M, La Vecchia C, Vatten L, Gavin A, Héry C, et al. Disparities in breast cancer mortality trends between 30 European countries: retrospective trend analysis of WHO mortality database. BMJ. 2010; 341: c3620.

3. Allemani C, Weir HK, Carreira H, Harewood R, Spika D, Wang X-S, et al Global surveillance of cancer survival 1995-2009: analysis of individual data for $25,676,887$ patients from 279 population-based registries in 67 countries (CONCORD-2). Lancet. 2015; 385: 977-1010.

4. Parry RV, Chemnitz JM, Frauwirth KA, Lanfranco AR, Braunstein I,
Kobayashi SV, et al. CTLA-4 and PD-1 receptors inhibit T-cell activation by distinct mechanisms. Mol Cell Biol. 2005; 25: 9543-9553.

5. Dong $\mathrm{H}$, Strome SE, Salomao DR, Tamura H, Hirano F, Flies DB, et al. Tumor-associated B7-H1 promotes T-cell apoptosis: a potential mechanism of immune evasion. Nat Med. 2002; 8: 793-800.

6. Reck M, Rodríguez-Abreu D, Robinson AG, Hui R, Csőszi T, Fülöp A, et al. Pembrolizumab versus Chemotherapy for PD-L1-Positive Non-Small-Cell Lung Cancer. N Engl J Med. 2016; 375: 1823-1833.

7. Mok TSK, Wu YL, Kudaba I, Kowalski DM, Cho BC, Turna HZ, et al. Pembrolizumab versus chemotherapy for previously untreated, PD-L1expressing, locally advanced or metastatic non-small-cell lung cancer (KEYNOTE-042): a randomised, open-label, controlled, phase 3 trial. Lancet. 2019; 393: 1819-1830.

8. Schachter J, Ribas A, Long GV, Arance A, Grob JJ, Mortier L, et al. Pembrolizumab versus ipilimumab for advanced melanoma: final overall survival results of a multicentre, randomised, open-label phase 3 study (KEYNOTE-006). Lancet. 2017; 390: 1853-1862.

9. Hodi FS, Chiarion-Sileni V, Gonzalez R, Grob JJ, Rutkowski P, Cowey CL, et al. Nivolumab plus ipilimumab or nivolumab alone versus ipilimumab alone in advanced melanoma (CheckMate 067): 4-year outcomes of a multicentre, randomised, phase 3 trial. Lancet Oncol. 2018; 19: 1480-1492.

10. Motzer RJ, Tannir NM, McDermott DF, Arén Frontera O, Melichar B, Choueiri TK, et al. Nivolumab plus Ipilimumab versus Sunitinib in Advanced Renal-Cell Carcinoma. N Engl J Med. 2018; 378: 1277-1290.

11. Powles T, Durán I, van der Heijden MS, Loriot Y, Vogelzang NJ, De Giorgi $U$, et al. Atezolizumab versus chemotherapy in patients with platinumtreated locally advanced or metastatic urothelial carcinoma (IMvigor211): a multicentre, open-label, phase 3 randomised controlled trial. Lancet. 2018; 391: 748-757.

12. Ferris RL, Blumenschein G Jr, Fayette J, Guigay J, Colevas AD, Licitra L, et al. Nivolumab for Recurrent Squamous-Cell Carcinoma of the Head and Neck. N Engl J Med. 2016; 375: 1856-1867.

13. Kandoth C, McLellan MD, Vandin F, Ye K, Niu B, Lu C, et al. Mutational landscape and significance across 12 major cancer types. Nature. 2013; 502: 333-339.

14. Kotoula V, Lakis S, Vlachos IS, Giannoulatou E, Zagouri F, Alexopoulou Z, et al. Tumor Infiltrating Lymphocytes Affect the Outcome of Patients with Operable Triple-Negative Breast Cancer in Combination with Mutated Amino Acid Classes. PLoS One. 2016; 11: e0163138.

15. Kitano A, Ono M, Yoshida M, Noguchi E, Shimomura A, Shimoi T, et al. Tumour-infiltrating lymphocytes are correlated with higher expression levels of PD-1 and PD-L1 in early breast cancer. ESMO Open. 2017; 2: e000150.

16. Nolan E, Savas P, Policheni AN, Darcy PK, Vaillant F, Mintoff CP, et al. Combined immune checkpoint blockade as a therapeutic strategy for BRCA1mutated breast cancer. Sci Transl Med. 2017; 9: eaal4922.

17. Denkert C, von Minckwitz G, Darb-Esfahani S, Lederer B, Heppner BI, Weber $\mathrm{KE}$, et al. Tumour-infiltrating lymphocytes and prognosis in different subtypes of breast cancer: a pooled analysis of 3771 patients treated with neoadjuvant therapy. Lancet Oncol. 2018; 19: 40-50.

18. Zhao J, Huang J. Breast cancer immunology and immunotherapy: targeting the programmed cell death protein-1/programmed cell death protein ligand-1. Chin Med J (Engl). 2020; 133: 853-862.

19. Dieci MV, Miglietta F, Guarneri V. Immune Infiltrates in Breast Cancer: Recent Updates and Clinical Implications. Cells. 2021; 10: 223.

20. Lotfinejad P, Kazemi T, Mokhtarzadeh A, Shanehbandi D, Jadidi Niaragh F, Safaei S, et al. PD-1/PD-L1 axis importance and tumor microenvironment immune cells. Life Sci. 2020; 259: 118297.

21. Penault-Llorca F, Viale G. Pathological and molecular diagnosis of triplenegative breast cancer: a clinical perspective. Ann Oncol. 2012; 23: vi19-22.

22. Dent R, Trudeau M, Pritchard KI, Hanna WM, Kahn HK, Sawka CA, et al. Triple-negative breast cancer: clinical features and patterns of recurrence. 
Clin Cancer Res. 2007; 13: 4429-4434.

23. Haffty BG, Yang Q, Reiss M, Kearney T, Higgins SA, Weidhaas J, et al. Locoregional relapse and distant metastasis in conservatively managed triple negative early-stage breast cancer. J Clin Oncol. 2006; 24: 5652-5657.

24. Caswell-Jin JL, Plevritis SK, Tian L, Cadham CJ, Xu C, Stout NK, et al. Change in Survival in Metastatic Breast Cancer with Treatment Advances: Meta-Analysis and Systematic Review. JNCI Cancer Spectr. 2018; 2: pky062.

25. Gobbini E, Ezzalfani M, Dieras V, Bachelot T, Brain E, Debled M, et al. Time trends of overall survival among metastatic breast cancer patients in the reallife ESME cohort. Eur J Cancer. 2018; 96: 17-24.

26. Yardley DA, Coleman R, Conte P, Cortes J, Brufsky A, Shtivelband M, et al. nab-Paclitaxel plus carboplatin or gemcitabine versus gemcitabine plus carboplatin as first-line treatment of patients with triple-negative metastatic breast cancer: results from the tnAcity trial. Ann Oncol. 2018; 29: 1763-1770.

27. Miles DW, Diéras V, Cortés J, Duenne AA, Yi J, O'Shaughnessy J. Firstline bevacizumab in combination with chemotherapy for HER2-negative metastatic breast cancer: pooled and subgroup analyses of data from 2447 patients. Ann Oncol. 2013; 24: 2773-2780.

28. Ali HR, Glont SE, Blows FM, Provenzano E, Dawson SJ, Liu B, et al. PD-L1 protein expression in breast ancer is rare, enriched in basal-like tumours and associated with infiltrating lymphocytes. Ann Oncol. 2015; 26: 1488-1493.

29. Sobral-Leite M, Van de Vijver K, Michaut M, van der Linden R, Hooijer GKJ, Horlings HM, et al. Assessment of PD-L1 expression across breast cancer molecular subtypes, in relation to mutation rate, BRCA1-like status, tumorinfiltrating immune cells and survival. Oncoimmunology. 2018; 7: e1509820.

30. Banerji S, Cibulskis K, Rangel-Escareno C, Brown KK, Carter SL, Frederick $\mathrm{AM}$, et al. Sequence analysis of mutations and translocations across breast cancer subtypes. Nature. 2012; 486: 405-409.

31. Nanda R, Chow LQ, Dees EC, Berger R, Gupta S, Geva R, et al Pembrolizumab in Patients With Advanced Triple-Negative Breast Cancer: Phase Ib KEYNOTE-012 Study. J Clin Oncol. 2016; 34: 2460-2467.

32. Emens LA, Cruz C, Eder JP, Braiteh F, Chung C, Tolaney SM, et al. Longterm Clinical Outcomes and Biomarker Analyses of atezolizumab Therapy for Patients With Metastatic Triple-Negative Breast Cancer: A Phase 1 Study. JAMA Oncol. 2019; 5: 74-82.

33. Adams S, Schmid P, Rugo HS, Winer EP, Loirat D, Awada A, et al. Pembrolizumab monotherapy for previously treated metastatic triple-negative breast cancer: cohort A of the phase II KEYNOTE-086 study. Ann Oncol. 2019; 30: 397-404.

34. Adams S, Loi S, Toppmeyer D, Cescon DW, De Laurentiis M, Nanda $\mathrm{R}$, et al. Pembrolizumab monotherapy for previously untreated, PD-L1positive, metastatic triple-negative breast cancer: cohort B of the phase II KEYNOTE-086 study. Ann Oncol. 2019; 30: 405-411.

35. Dirix LY, Takacs I, Jerusalem G, Nikolinakos P, Arkenau HT, Forero-Torres $A$, et al. Avelumab, an anti-PD-L1 antibody, in patients with locally advanced or metastatic breast cancer: a phase $1 \mathrm{~b}$ JAVELIN Solid Tumor study. Breast Cancer Res Treat. 2018; 167: 671-686.

36. Winer EP, Lipatov O, Im SA, Goncalves A, Muñoz-Couselo E, Seok Lee K, et al. Pembrolizumab versus investigator-choice chemotherapy for metastatic triple-negative breast cancer (KEYNOTE-119): a randomised, open-label, phase 3 trial. Lancet Oncol. 2021; 22: 499-511.

37. Kroemer G, Galluzzi L, Kepp O, Zitvogel L. Immunogenic cell death in cance therapy. Annu Rev Immunol. 2013; 31: 51-72.

38. Cortes J, Cescon DW, Rugo HS, Nowecki Z, Im SA, Md Yusof M, et al. Pembrolizumab plus chemotherapy versus placebo plus chemotherapy for previously untreated locally recurrent inoperable or metastatic triple-negative breast cancer (KEYNOTE-355): a randomised, placebo-controlled, doubleblind, phase 3 clinical trial. Lancet. 2020; 396: 1817-1828.

39. Rugo H, Schmid ., Cescon D, Nowecki Z, Im SA., Md Yusof M, et al. Additional efficacy endpoints from the phase 3 KEYNOTE-355 study of pembrolizumab plus chemotherapy vs. placebo plus chemotherapy as first-line therapy for locally recurrent inoperable or metastatic triple-negative breast cancer.
Proceedings of San Antonio Breast Cancer Symposium. 2020,

40. Tolaney SM, Kalinsky K, Kaklamani VG, D'Adamo DR, Aktan G, Tsai ML, et al. Eribulin Plus Pembrolizumab in Patients with Metastatic Triple-Negative Breast Cancer (ENHANCE 1): A Phase Ib/ll Study. Clin Cancer Res. 2021.

41. Adams S, Diamond JR, Hamilton E, Pohlmann PR, Tolaney SM, Chang VW, et al. Atezolizumab Plus nab-Paclitaxel in the Treatment of Metastatic TripleNegative Breast Cancer With 2-Year Survival Follow-up: A Phase 1b Clinical Trial. JAMA Oncol. 2019; 5: 334-342.

42. Schmid P, Adams S, Rugo HS, Schneeweiss A, Barrios $\mathrm{CH}$, Iwata $\mathrm{H}$, et al. Atezolizumab and Nab-Paclitaxel in Advanced Triple-Negative Breast Cancer. N Engl J Med. 2018; 379: 2108-2121.

43. Schmid P, Rugo HS, Adams S, Schneeweiss A, Barrios $\mathrm{CH}$, Iwata $\mathrm{H}$, et al. Atezolizumab plus nab-paclitaxel as first-line treatment for unresectable, locally advanced or metastatic triple-negative breast cancer (IMpassion130): updated efficacy results from a randomised, double-blind, placebo-controlled, phase 3 trial. Lancet Oncol. 2020; 21: 44-59.

44. Miles D, Gligorov J, André F, Cameron D, Schneeweiss A, Barrios CH, et al. Primary results from IMpassion131, a double-blind placebo-controlled randomised phase III trial of first-line paclitaxel (PAC) \pm atezolizumab (atezo) for unresectable locally advanced/metastatic triple-negative breast cancer (mTNBC). Annals of Oncology. 2020; 31: S1142-S1215.

45. Berry DA, Cirrincione C, Henderson IC, Citron ML, Budman DR, Goldstein LJ, et al. Estrogen-receptor status and outcomes of modern chemotherapy for patients with node-positive breast cancer. JAMA. 2006; 295: 1658-1667.

46. Burstein HJ, Curigliano G, Loibl S, Dubsky P, Gnant M, Poortmans P, et al. Estimating the benefits of therapy for early-stage breast cancer: the St. Gallen International Consensus Guidelines for the primary therapy of early breast cancer 2019. Ann Oncol. 2019; 30: 1541-1557.

47. Cardoso F, Kyriakides S, Ohno S, Penault-Llorca F, Poortmans P, Rubio IT, et al. Early breast cancer: ESMO Clinical Practice Guidelines for diagnosis, treatment and follow-upt. Ann Oncol. 2019; 30: 1194-1220.

48. Pusztai L, Foldi J, Dhawan A, DiGiovanna MP, Mamounas EP. Changing frameworks in treatment sequencing of triple-negative and HER2-positive, early-stage breast cancers. Lancet Oncol. 2019; 20: e390-e396.

49. Cortazar P, Zhang L, Untch M, Mehta K, Costantino JP, Wolmark N, et al. Pathological complete response and long-term clinical benefit in breast cancer: the CTNeoBC pooled analysis. Lancet. 2014; 384: 164-172.

50. Liedtke C, Mazouni C, Hess KR, André F, Tordai A, Mejia JA, et al. Response to neoadjuvant therapy and long-term survival in patients with triple-negative breast cancer. J Clin Oncol. 2008; 26: 1275-1281.

51. Poggio F, Bruzzone M, Ceppi M, Pondé NF, La Valle G, Del Mastro L, et al. Platinum-based neoadjuvant chemotherapy in triple-negative breast cancer: a systematic review and meta-analysis. Ann Oncol. 2018; 29: 1497-1508.

52. Guidance for industry. pathological complete response in neoadjuvant treatment of high- risk early-stage breast cancer: use as an endpoint to support accelerated approval. Silver Spring, MD: Food and Drug Administration. 2014

53. The role of the pathological complete response as an endpoint in neoadjuvant breast cancer studies. London: European Medicines Agency. 2014.

54. Nanda R, Liu MC, Yau C, Shatsky R, Pusztai L, Wallace A, et al. Effect of pembrolizumab Plus Neoadjuvant Chemotherapy on Pathologic Complete Response in Women With Early-Stage Breast Cancer: An Analysis of the Ongoing Phase 2 Adaptively Randomized I-SPY2 Trial. JAMA Oncol. 2020; 6: 676-684.

55. Schmid P, Salgado R, Park YH, Muñoz-Couselo E, Kim SB, Sohn J et al. Pembrolizumab plus chemotherapy as neoadjuvant treatment of high-risk early-stage triple-negative breast cancer: results from the phase $1 \mathrm{~b}$ openlabel, multicohort KEYNOTE-173 study. Ann Oncol. 2020; 31: 569-581.

56. Schmid P, Cortes J, Pusztai L, McArthur H, Kümmel S, Bergh J, et al. Pembrolizumab for Early Triple-Negative Breast Cancer. N Engl J Med. 2020; 382: 810-821.

57. Mittendorf EA, Zhang $\mathrm{H}$, Barrios $\mathrm{CH}$, Saji S, Jung $\mathrm{KH}$, Hegg R, et al. 
Neoadjuvant atezolizumab in combination with sequential nab-paclitaxel and anthracycline-based chemotherapy versus placebo and chemotherapy in patients with early-stage triple-negative breast cancer (IMpassion031): a randomised, double-blind, phase 3 trial. Lancet. 2020; 396: 1090-1100.

58. Loibl S, Untch M, Burchardi N, Huober J, Sinn BV, Blohmer JU, et al A randomised phase II study investigating durvalumab in addition to an anthracycline taxane-based neoadjuvant therapy in early triple-negative breast cancer: clinical results and biomarker analysis of GeparNuevo study. Ann Oncol. 2019; 30: 1279-1288.

59. Gianni L, Huang CS, Egle D, Bermejo B, Zamgni C, Thill M, et al. Pathologic complete response $(\mathrm{pCR})$ to neoadjuvant treatment with or without atezolizumab in triple negative, early high-risk and locally advanced breast cancer. NeoTRIPaPDL1 Michelangelo randomized study. Proceedings of San Antonio Breast Cancer Symposium. 2019

60. Voorwerk L, Slagter M, Horlings HM, Sikorska K, van de Vijver KK, de Maaker $\mathrm{M}$, et al. Immune induction strategies in metastatic triple-negative breast cancer to enhance the sensitivity to PD-1 blockade: the TONIC trial. Nat Med 2019; 25: 920-928.

61. Maximov PY, Lee TM, Jordan VC. The discovery and development of selective estrogen receptor modulators (SERMs) for clinical practice. Curr Clin Pharmacol. 2013; 8: 135-155

62. Mittendorf EA, Philips AV, Meric-Bernstam F, Qiao N, Wu Y, Harrington S, et al. PD-L1 expression in triple-negative breast cancer. Cancer Immunol Res. 2014; 2 : 361-370.

63. Denkert C, von Minckwitz G, Darb-Esfahani S, Lederer B, Heppner BI, Weber $\mathrm{KE}$, et al. Tumour-infiltrating lymphocytes and prognosis in different subtypes of breast cancer: a pooled analysis of 3771 patients treated with neoadjuvant therapy. Lancet Oncol. 2018; 19: 40-50.

64. Luen S, Virassamy B, Savas P, Salgado R, Loi S. The genomic landscape of breast cancer and its interaction with host immunity. Breast. 2016; 29: 241 250

65. Rugo HS, Delord JP, Im SA, Ott PA, Piha-Paul SA, Bedard PL, et al. Safety and Antitumor Activity of pembrolizumab in Patients with Estrogen ReceptorPositive/Human Epidermal Growth Factor Receptor 2-Negative Advanced Breast Cancer. Clin Cancer Res. 2018; 24: 2804-2811.

66. Tolaney SM, Barroso-Sousa R, Keenan T, Li T, Trippa L, Vaz-Luis I, e al. Effect of Eribulin With or Without pembrolizumab on Progression-Free Survival for Patients With Hormone Receptor-Positive, ERBB2-Negative Metastatic Breast Cancer: A Randomized Clinical Trial. JAMA Oncol. 2020; 6: 1598-1605.

67. Dawood S, Broglio K, Buzdar AU, Hortobagyi GN, Giordano SH. Prognosis of women with metastatic breast cancer by HER2 status and trastuzumab treatment: an institutional-based review. J Clin Oncol. 2010; 28: 92-98.

68. von Minckwitz G, Procter M, de Azambuja E, Zardavas D, Benyunes M Viale $\mathrm{G}$ et al. Adjuvant Pertuzumab and Trastuzumab in Early HER2-Positive Breast Cancer. N Engl J Med. 2017; 377: 122-131.

69. Swain SM, Baselga J, Kim SB, Ro J, Semiglazov V, Campone M et al Pertuzumab, trastuzumab, and docetaxel in HER2-positive metastatic breast cancer. N Engl J Med. 2015; 372: 724-734.

70. Diéras V, Miles D, Verma S, Pegram M, Welslau M, Baselga J, et al. Trastuzumab emtansine versus capecitabine plus lapatinib in patients with previously treated HER2-positive advanced breast cancer (EMILIA): a descriptive analysis of final overall survival results from a randomised, openlabel, phase 3 trial. Lancet Oncol. 2017; 18: 732-742

71. Stagg J, Loi S, Divisekera U, Ngiow SF, Duret H, Yagita H, et al. Anti-ErbB-2 $\mathrm{mAb}$ therapy requires type I and II interferons and synergizes with anti-PD-1 or anti-CD137 mAb therapy. Proc Natl Acad Sci USA. 2011; 108: 7142-7147.

72. Chia S, Bedard PL, Hilton J, Amir E, Gelmon K, Goodwin R, et al. A Phase Ib Trial of durvalumab in Combination with Trastuzumab in HER2-Positive
Metastatic Breast Cancer (CCTG IND.229). Oncologist. 2019; 24: 1439-1445.

73. Loi S, Giobbie-Hurder A, Gombos A, Bachelot T, Hui R, Curigliano G, et al. Pembrolizumab plus trastuzumab in trastuzumab-resistant, advanced, HER2-positive breast cancer (PANACEA): a single-arm, multicentre, phase 1b-2 trial. Lancet Oncol. 2019; 20: 371-382.

74. Emens LA, Esteva FJ, Beresford M, Saura C, De Laurentiis M, Kim SB, et al. Trastuzumab emtansine plus atezolizumab versus trastuzumab emtansine plus placebo in previously treated, HER2-positive advanced breast cancer (KATE2): a phase 2, multicentre, randomised, double-blind trial. Lancet Oncol. 2020; 21: 1283-1295.

75. Hamilton E, Shapiro CL, Petrylak D, Boni V, Martin M, Del Conte G, et al. Trastuzumab deruxtecan (T-DXd; DS-8201) with nivolumab in patients with HER2-expressing, advanced breast cancer: A 2-part, phase 1b, multicenter, open-label study. Proceeding for San Antonio Breast Cancer Symposium. 2020.

76. Wang S, Sun K, Xiao Y, Feng B, Mikule K, Ramaswamy S, et al. Evaluation of niraparib in combination with anti-PD1/anti-PD-L1 in preclinical models. Cancer Res. 2018; 78: abstract 1724.

77. Vinayak S, Tolaney SM, Schwartzberg L, Mita M, McCann G, et al. OpenLabel Clinical Trial of Niraparib Combined With pembrolizumab for Treatment of Advanced or Metastatic Triple-Negative Breast Cancer. JAMA Oncol. 2019; 5: 1132-1140.

78. Kaneda MM, Messer KS, Ralainirina N, Li H, Leem CJ, Gorjestani S, et al. $\mathrm{PI}$ Kgamma is a molecular switch that controls immune suppression. Nature. 2016; 539: 437-442.

79. Schmid P, Loirat D, Savas P, Espinosa E, Boni V, Italiano A et al. Phase lb study evaluating a triple combination of ipatasertib (IPAT), atezolizumab (atezo), and paclitaxel (PAC) or nab-PAC as first-line (1L) therapy for locally advanced/metastatic triple-negative breast cancer (TNBC). Proceeding for AACR Annual Meeting. 2019

80. Rugo HS, Loi S, Adams S, Schmid P, Schneeweiss A, Barrios $\mathrm{CH}$, et al. Performance of PD-L1 immunohistochemistry (IHC) assays in unresectable locally advanced or metastatic triple-negative breast cancer (mTNBC): Posthoc analysis of IMpassion130. Annals of Oncology. 2019; 30: v851-v934.

81. Barroso-Sousa R, Keenan TE, Pernas S, Exman P, Jain E, Garrido-Castro $\mathrm{AC}$, et al. Tumor Mutational Burden and PTEN Alterations as Molecular Correlates of Response to PD-1/L1 Blockade in Metastatic Triple-Negative Breast Cancer. Clin Cancer Res. 2020; 26: 2565-2572.

82. Kwapisz D. Pembrolizumab and atezolizumab in triple-negative breast cancer. Cancer Immunol Immunother. 2021; 70: 607-617.

83. Loi S, Winer W, Lipatov O, Im S-A, Goncalves A, Cortes J, et al. Relationship between tumor-infiltrating lymphocytes (TILs) and outcomes in the KEYNOTE-119 study of pembrolizumab vs chemotherapy for previously treated metastatic triple-negative breast cancer (mTNBC). Proceeding for San Antonio Breast Cancer Symposium. 2020.

84. Emens LA, Loi S, Rugo HS, Schneeweiss A, Diéras $V$, Iwata $H$, et al. IMpassion130: Efficacy in immune biomarker subgroups from the global, randomized, double-blind, placebo-controlled, phase III study of atezolizumab + nab-paclitaxel in patients with treatment-naïve, locally advanced or metastatic triple-negative breast cancer. Proceeding for San Antonio Breast Cancer Symposium. 2019.

85. Loibl S, Sinn B, Karn T, Untch M, Sinn HP, Weber KE, et al. Exome analysis of oncogenic pathways and Tumor Mutational Burden (TMB) in Triple-Negative Breast Cancer (TNBC): Results of the translational biomarker program of the neoadjuvant double-blind placebo controlled GeparNuevo trial. Journal of Clinical Oncology. 2019; 37: 509-509.

86. Byrne A, Savas P, Sant S, Li R, Virassamy B, Luen SJ, et al. Tissue-resident memory $T$ cells in breast cancer control and immunotherapy responses. Nat Rev Clin Oncol. 2020; 17: 341-348. 